OPEN ACCESS

Edited by:

Weiwei Wu,

Xidian University, China

Reviewed by:

Zhongchang Wang International Iberian Nanotechnology Laboratory (INL), Portuga

Ming-Guo Ma,

Beijing Forestry University, China Keng $X u$,

Jiangxi Normal University, China

*Correspondence:

Weigen Chen

weigench@cqu.edu.cn Yu Wang

wangyu@succ.edu.cn

Specialty section: This article was submitted to Nanoscience,

a section of the journal

Frontiers in Chemistry

Received: 31 December 2019

Accepted: 26 February 2020

Published: 07 April 2020

Citation:

Tang S, Chen W, Zhang H, Song Z,

Li Y and Wang Y (2020) The

Functionalized Single-Walled Carbon

Nanotubes Gas Sensor With Pd

Nanoparticles for Hydrogen Detection

in the High-Voltage Transformers.

Front. Chem. 8:174.

doi: 10.3389/fchem.2020.00174

\section{The Functionalized Single-Walled Carbon Nanotubes Gas Sensor With Pd Nanoparticles for Hydrogen Detection in the High-Voltage Transformers}

\author{
Sirui Tang ${ }^{1}$, Weigen Chen ${ }^{1 *}$, He Zhang ${ }^{1}$, Zihao Song ${ }^{1}$, Yanqiong $L i^{2}$ and Yu Wang ${ }^{3 *}$ \\ 1 State Key Laboratory of Power Transmission Equipment and System Security and New Technology, Chongqing University, \\ Chongqing, China, ${ }^{2}$ School of Electronic and Electrical Engineering, Chongqing University of Arts and Sciences, Chongqing, \\ China, ${ }^{3}$ Shanghai Urban Construction Vocational College, Shanghai, China
}

Single-walled carbon nanotubes (SWCNTs) have been widely discussed and applied as novel gas sensing nanomaterials. Hydrogen is one of the remarkable fault characteristic gases in high-voltage oil-paper insulated transformers. In this paper, 3.07 wt\% Pd nanoparticles (NPs) were used to decorate SWCNTs. The unloaded, the carboxylated, and the Pd-doped SWCNTs were fabricated into three planar gas sensors, and their gas sensing properties to hydrogen were studied. Gas sensing mechanism was analyzed. Results show that the optimal operating temperature of a Pd-doped SWCNTs-based gas sensor is $125^{\circ} \mathrm{C}$ lower than that of the unloaded SWCNTs-based gas sensor, and it shows the highest gas sensing response value. This is attributed to the decreasing work function of $\mathrm{Pd}$, which reduces the hole carries in the nanotubes.

Keywords: single-walled carbon nanotubes, Pd-doped, hydrogen detection, gas sensing properties, high-voltage transformers

\section{INTRODUCTION}

Novel gas sensing materials were discovered and have been studied over the past five decades. A number of works on the improvement of microstructures, structures, and sensing properties of gas sensing materials have been done by researchers. Nanomaterials, such as nanofibers, nanowires, carbon nanotubes (CNTs), and nanoparticles, are the main focus of this research. In a study by Zhou et al. (2017a,b), the highly porous $\mathrm{NiO}$ nanodisks (NiO-NDs) and its synthesis, characterization, and sensing applications to alcohol were analyzed. In the reference (Zhou et al., 2018), the 1D hierarchical p-n heterostructured $\mathrm{Mn}_{3} \mathrm{O}_{4} / \mathrm{SnO}_{4}$ hybrid materials (HMs) was synthesized by Zhou et al., and the sensing results to acetone indicates the perfect gas sensing performance of hybrid materials.

Due to CNTs fullerene structure and its large surface area, and the excellent electrical, mechanical, and thermal properties they have, CNTs have been one of the most widely studied gas sensing materials in the past two decades (Chen et al., 2001; Rana et al., 2017; Zaporotskova et al., 2017). Single-walled carbon nanotubes (SWCNTs) and multi-walled carbon nanotubes (MWCNTs) are the two main types of CNTs. The microstructure of SWCNTs and MWCNTs are somehow the same, that is, they consist of a rolled-up single sheet of a layer of graphene. However, MWCNTs are 
composed of concentric tubes of graphene fitted inside each other (Pitroda et al., 2016; Beitollahi et al., 2018; Han et al., 2019). It is confirmed that adsorption of electron withdrawing (e.g., $\mathrm{NO}_{2}, \mathrm{O}_{2}$ ) or donating (like $\mathrm{NH}_{3}$ ) molecules on SWCNTs will cause the charge transfer between the nanotubes and molecules (Kong et al., 2001). Compared with other gas sensing materials, like MOS, CNTs-especially single-walled carbon nanotubeshave remarkable properties. For example, they have the highest Young's modulus, highest thermal conductivity, ballistic electron transport, and a high aspect ratio structure. What's more, CNTs are a more stable electrode material than other gas sensing materials due to its lower probability to be reduced or oxidized during a substantial range of potentials (Robertson, 2004). In the reference (Naje et al., 2016), the detection of $\mathrm{NO}_{2}$ using SWCNTs and MWCNTs on porous silicon wafers was done by Naje et al. The $\mathrm{NO}_{2}$ gas sensing performance of SWCNTs and MWCNTs vary at temperatures ranging from 25 to $250^{\circ} \mathrm{C}$, and it shows that equal sensitivity can be reached with a higher temperature for SWCNTs compared to MWCNTs, while the highest response of SWCNTs (79.8\%) is higher than MWCNTs (59.6\%) at their optimum temperature $\left(150^{\circ} \mathrm{C}\right.$ for SWCNTs, $200^{\circ} \mathrm{C}$ for MWCNTs). The trace level detection of $\mathrm{NH}_{3}$ and $\mathrm{NO}_{2}$ at room temperature via randomly oriented SWCNTs, which is grown by PECVE technique at $650^{\circ} \mathrm{C}$, were realized by Lone et al. (2018). Results show the quick response and recovery characteristics of both $\mathrm{NH}_{3}$ and $\mathrm{NO}_{2}$. Additionally, the gas sensing abilities of unloaded SWCNTs to $\mathrm{N}_{2} \mathrm{O}_{4}$ (Dai et al., 1999), $\mathrm{O}_{2}$ (Kong et al., 1998), $\mathrm{CO}_{2}$ (Yoon et al., 2018), and $\mathrm{CH}_{4}$ (Poonia et al., 2015) are widely discussed.

High-voltage oil-paper insulated transformers play an essential role in power transmission, but during the longterm operation, due to oxidation, pollution, and excessive inner temperature, the transformer insulation oil will be degraded and decomposed, producing traces of characteristic gases dissolving in the oil. Hydrogen is one of the main characteristic gases reflecting overheat fault and discharge

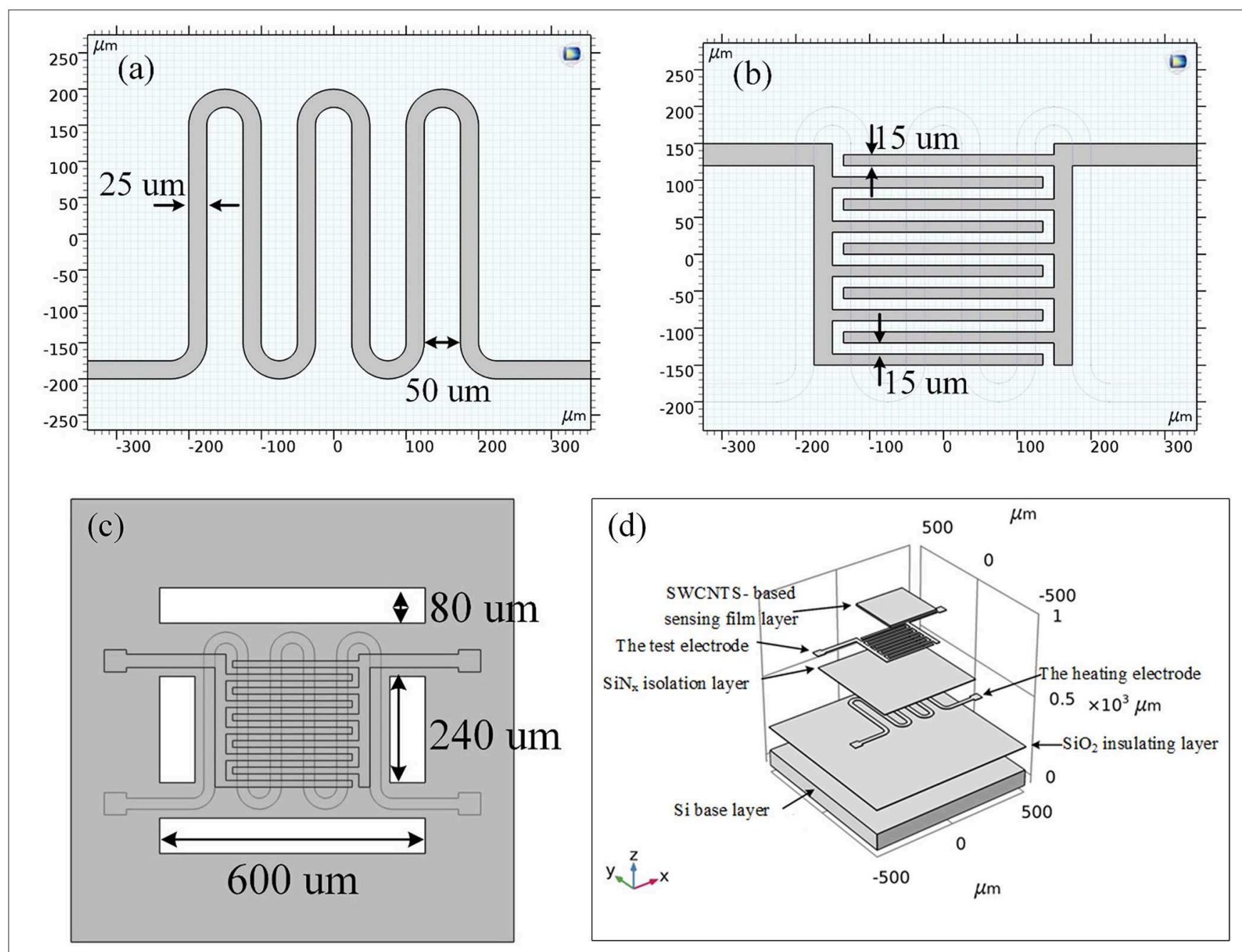

FIGURE 1 | (a-c) The 2D structure of the planar gas sensor. (d) The 3D structure of the planar gas sensor. 
fault in the oil-immersed transformer (International Standard IEC 60599: 2015, 2015). Gas sensing nanomaterials such as $\mathrm{SnO}_{2}, \mathrm{ZnO}, \mathrm{WO}_{3}$, and $\mathrm{MoS}_{2}$, as well as their functionalized derivatives, have been applied in studies on characteristic gases detecting of high-voltage transformers (Tang et al., 2017; Zhou et al., 2018; Wang et al., 2019; Wei et al., 2019). More importantly, Zhang et al. found that CNTs are well-performed gas sensing materials for gas detection of high-voltage electrical equipment (Zhang et al., 2017). However, it was reported in the study Kong et al. (2001) that nanotubes show a poor sensing response to some gas molecules. Instead, geometrical optimizations of SWCNTs with and without doped metals and their gas adsorption structures were studied based on computational methodology by applying restricted density functional theory (DFT) by Tabtimsai et al. (2011). Calculation and simulation indicated that decorating SWCNTs with $\mathrm{Pd}$ nanoparticles can effectively enhance the gas sensing performance to $\mathrm{NO}_{2}, \mathrm{NH}_{3}, \mathrm{H}_{2} \mathrm{O}$, and $\mathrm{H}_{2}$. The hydrogen atoms dissociated from the hydrogen molecules have smaller adsorption energy and dissociation energy on the Pd cluster. As an electron donor, Pd clusters can quickly dissociate hydrogen atoms, electron acceptors, and accelerate electron transfer in gas-sensitive materials.

In this paper, the carboxylated and $3.07 \mathrm{wt} \%$ Pd-doped SWCNTs-based nanomaterials were synthesized based on the unloaded SWCNTs-based nanomaterials. Three SWCNTsbased nanomaterials (the unloaded, the carboxylated, and the Pd-doped) were fabricated into corresponding planar gas sensors. Gas sensing properties including the temperature characteristics, the concentration characteristics, the linearity, detecting limitation, and the response and recovery time characteristics of three SWCNTs-based gas sensors to hydrogen were studied. Gas sensing mechanisms were analyzed. In the results, Pd-doped SWCNTs-base gas sensor presents the best gas sensing performance to hydrogen. This study can provide a novel solution to the issue of characteristic gases detection in high-voltage oil-paper insulated transformers.

\section{MATERIALS AND METHODS Preparation of SWCNTs-Based Nanomaterials}

The unloaded SWCNTs-based nanomaterials were made by Timesnano of Chengdu Organic Chemicals Co. Ltd., Chinese Academy of Sciences. The outer Diameter (OD) of the unloaded SWCNTs-based gas sensing nanomaterials is $1-3 \mathrm{~nm}$, and the purity is all higher than $90 \mathrm{wt} \%$. The length of nanomaterials is about 50 microns. Concentrated hydrochloric acid, concentrated sulfuric acid, concentrated nitric acid, isopropanol, and ammonia used in the preparation process were purchased from Chongqing Chuandong Chemical Co., Ltd. (China), and all were of analytical grade. Palladium chloride $\left(\mathrm{PdCl}_{2}\right)$ provided a source of palladium. Deionized water was also used.

The processes of preparation of SWCNTs-based nanomaterials are classified into three steps:

(1) Purification of the unloaded SWCNTs

Six hundred milligram of unloaded SWCNTs were added to $300 \mathrm{ml}$ of concentrated hydrochloric acid and ultrasonically cleaned for $30 \mathrm{~min}$. Five hundred milligram of deionized water was used to ultrasonically clean the mixture for another $30 \mathrm{~min}$. The cleaned mixture was placed in a drying box and dried at $150^{\circ} \mathrm{C}$ for $10 \mathrm{~h}$. Purified unloaded SWCNTs were obtained.

(2) Acidification of the purified SWCNTs

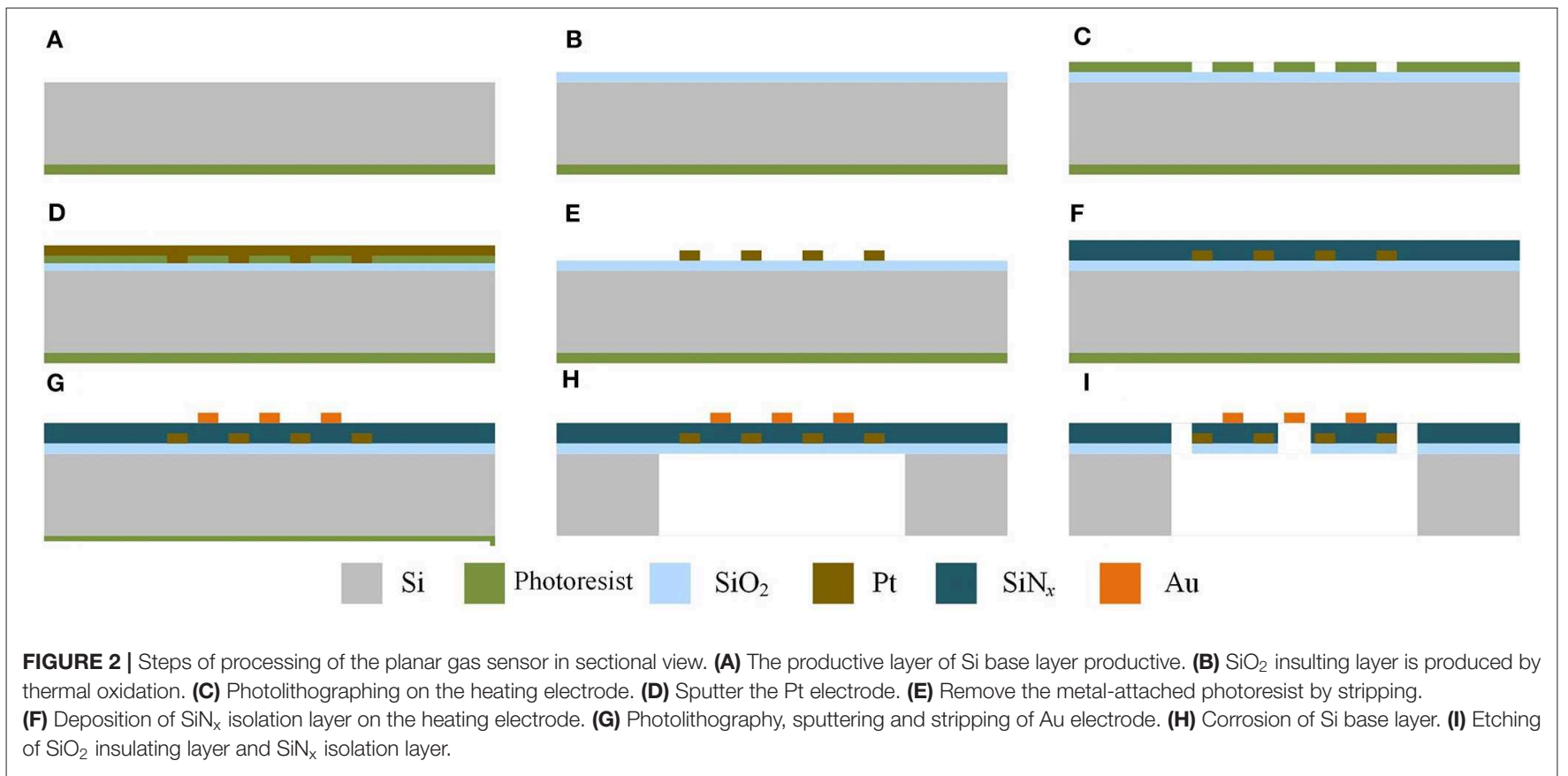




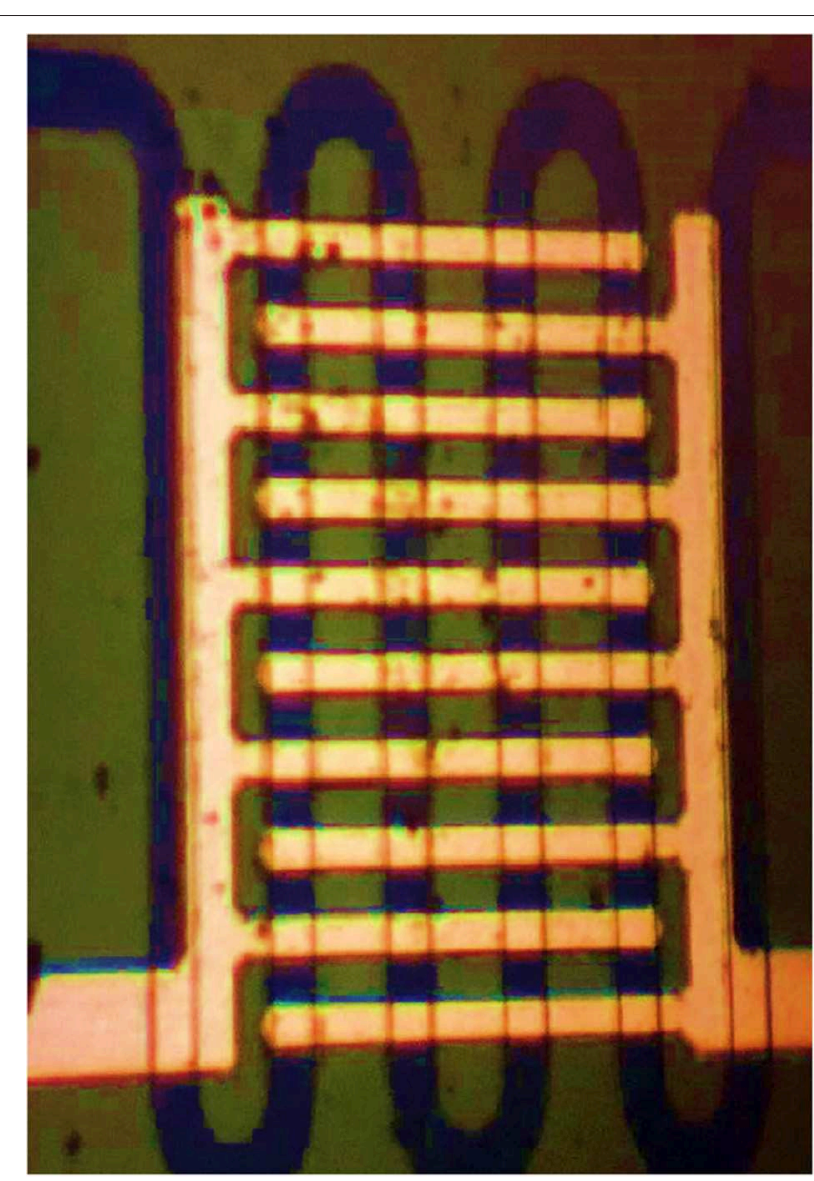

FIGURE 3 | The planar gas sensor.

Four hundred milligram of purified unloaded SWCNTs were added to $50 \mathrm{ml}$ of concentrated sulfuric acid and $20 \mathrm{ml}$ of concentrated nitric acid, and heated and stirred at $80^{\circ} \mathrm{C}$ for $4 \mathrm{~h}$. After the mixture was cooled down, it was diluted with $60 \mathrm{ml}$ deionized water. The mixture was filtered through a microporous membrane with a pore diameter of 0.45 microns, and washed repeatedly with deionized water until $\mathrm{pH}=7$. After drying at $80^{\circ} \mathrm{C}$ for $12 \mathrm{~h}$, acidified SWCNTs were obtained.

(3) Functionalization of the acidified SWCNTs

Two hundred milligram of SWCNT was dissolved in $50 \mathrm{ml}$ of isopropanol and sonicated for $20 \mathrm{~min}$ to improve the dispersibility of SWCNTs. $10.31 \mathrm{mg}$ of $\mathrm{PdCl}_{2}$ was dissolved in $3 \mathrm{ml}$ of ammonia water. $\mathrm{PdCl}_{2}$ solution was added to the SWCNTs/isopropanol mixed solution drop by drop, and stirred at a high speed for $2 \mathrm{~h}$. The obtained suspension was put in a drying box and dried at $80^{\circ} \mathrm{C}$ for $2 \mathrm{~h}$. The obtained powder was put in a calciner at $600^{\circ} \mathrm{C}$ for $2 \mathrm{~h} .3 .07 \mathrm{wt} \% \mathrm{Pd}$-doped SWCNTs-based nanomaterials were synthesized successfully.

The microstructure of the nanomaterials was characterized by Scanning Electron Microscopy (SEM) (SU8020, HITACHI, Japan) and Transmission Electron Microscopy (TEM) (JEM2000EX, JEOL, Japan).

\section{Fabricated of SWCNTs-Based Gas Sensors}

Planar gas sensors are applied in this paper. The structure of planar gas sensors is shown in Figure 1. The planar gas sensor is mainly composed of a Pt heating electrode, a Si base layer, an Au gas sensing electrode, and a SWCNTS-based sensing film layer. The size of the testing area is $300 \mu \mathrm{m} \times 300 \mu \mathrm{m}$. The hardware structure of the gas sensor is fabricated by Zhengzhou Winsen Electronics Technology CO., Ltd by processes mainly including oxidation, photolithography, sputtering, stripping, deposition, and etching, which are presented in Figure 2.

The planar sensor array is shown in Figure 3. The n-type (110) crystal face double polished silicon wafer (thickness is about $300 \mu \mathrm{m}$, and conductivity is $0.001-0.1 \mathrm{~S} / \mathrm{m}$ ) is used. In this paper, three SWCNTs-based sensing nanomaterials are coated by the droplet guiding method. After fully grinding the appropriate number of SWCNTs-based nanomaterials, it was dissolved in absolute ethanol for $1 \mathrm{~h}$ to obtain the corresponding dispersion, which was then dried at $400^{\circ} \mathrm{C}$ to get ultrafine powder. The deionized water droplets were applied to the sensor unit testing area using a micro syringe, and the SWCNTs-based nanomaterial powder was carefully applied to the droplets. After drying for $8 \mathrm{~h}$, the powder was closely attached to the testing area, and three SWCNTs-based planar gas sensors were fabricated successfully.

\section{Gas Sensing Test Methods}

The experimental platform consists of air source, RSC2000A automatic gas mixing system (Beijing JS Co.), and CGS-8 intelligent gas sensitivity analysis system (Beijing Elite Tech Co., China), which is presented in Figure 4. The detection of the electrical signals of the gas sensor is mainly realized by the classical resistor divider principle, and the test circuit is shown in Figure 5, where $R_{L}$ is the adjustable load resistance and $R_{S}$ is the resistance at both ends of the test electrode. $R_{H}$ is the resistance of the heating electrode of the sensing unit. $V_{H}$ and $V_{S}$ is the heating voltage and test voltage, respectively. During the test, $R_{S}$ will vary with the change of condition and can be calculated by the principle of voltage division.

The sensor response values defined here are as follows (Wang et al., 2010):

$$
R_{s}=\left(R_{\text {gas }}-R_{\text {air }}\right) / R_{\text {air }}
$$

Specific steps are as follows:

1. The gas sensor is placed in a closed air chamber filled with pure air $(20 \mathrm{~L})$, and the sensing signal $\left(R_{s}\right)$ is recorded by CGS- 8 system during the test.

2. The determined concentrations of hydrogen are injected by RSC2000-A system (gas flow: $250 \mathrm{sccm}$ ). The resistance value is recorded after the signal is stable.

3. The air chamber is opened through the fume hood and the resistance made to return to the initial value $R_{\text {air }}$.

4. Step (2) and step (3) are repeated.

5. The recorded data is saved and further experiments can be proceeded with.

Experiments were all carried out at a temperature of $28^{\circ} \mathrm{C}$ and at $60 \%$ humidity. 


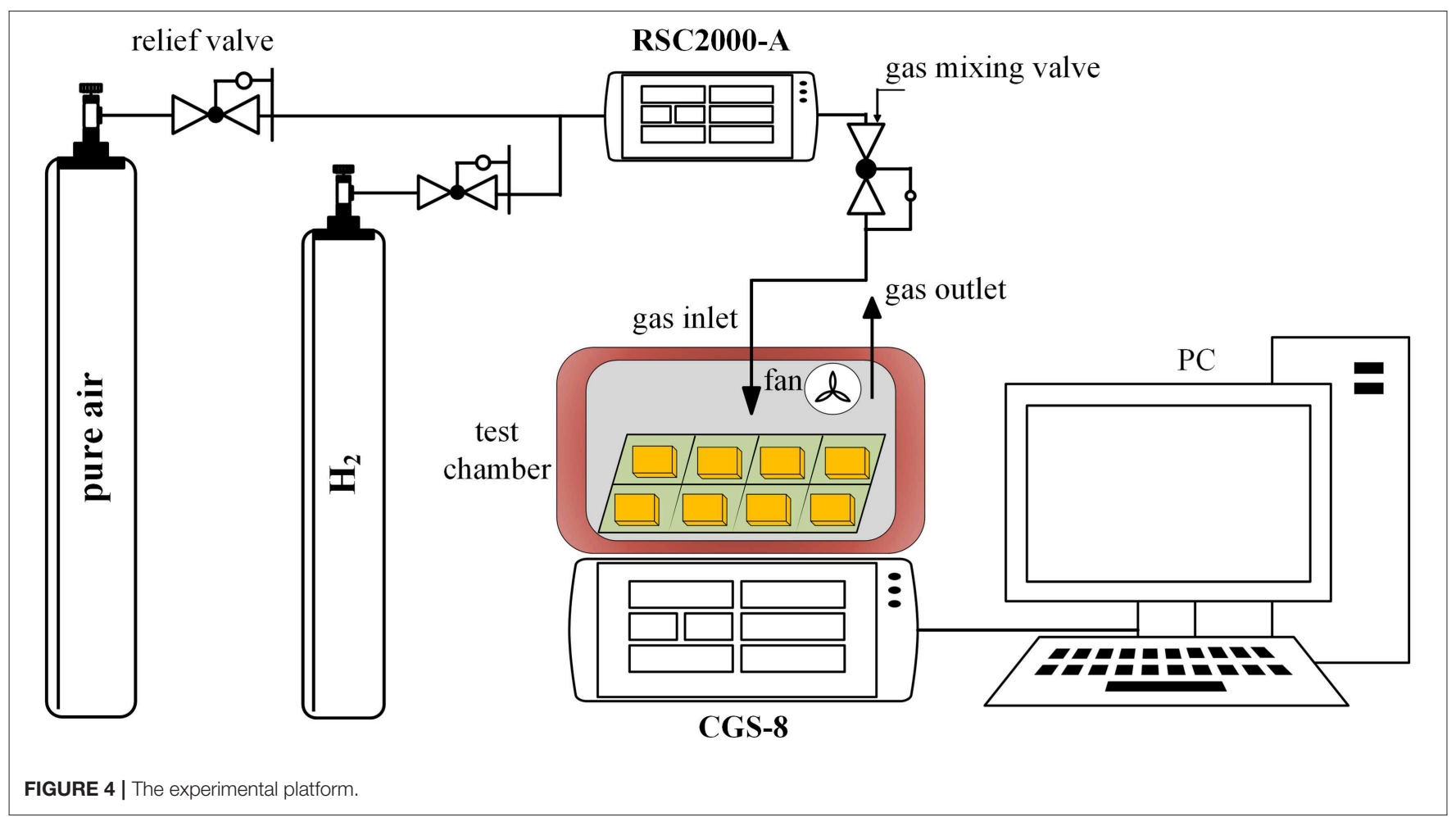

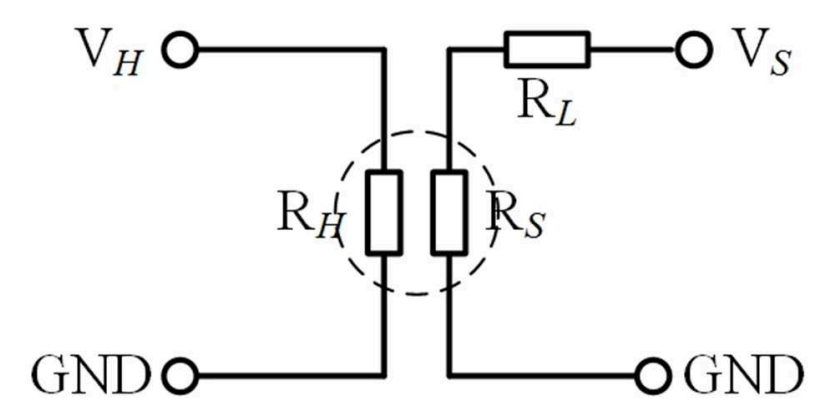

FIGURE 5 | The test circuit.

\section{RESULTS AND DISCUSSIONS}

\section{Morphology}

Figure 6 shows the SEM and TEM images of the unloaded, the carboxylated, and the Pd-doped SWCNTs. From the SEM images, the three kinds of SWCNTs are all intertwined and woven into a mesh, and the morphology of the SWCNTs after functioning has not changed much. However, in Figures $\mathbf{6 b}, \mathbf{c}$, the carboxylated and the Pd-doped SWCNTs are shorter in length and more distributed compared to the unloaded SWCNTs in Figure 6a. In the TEM images, all three SWCNTs are curved and tubular, and the basic structure has not been functionally damaged.

After acidification treatment, SWCNTs were oxidized under the action of concentrated sulfuric acid and concentrated nitric acid. Oxygen atoms released by concentrated nitric acid attacked carbon nanotubes, especially the defects at the ends and on the tube walls. Because carbon atoms are not stable six-membered rings in SWCNTs and are in a metastable state, SWCNTs will break in places where the nanotube curvature is large. Thus, the length of nanotubes becomes shorter, and nanotubes gradually disperse.

\section{Gas Sensing Properties}

The temperature characteristics, concentration characteristics (including linearity and detecting limitation), response and recovery time characteristics of the unloaded, the carboxylated, and the Pd-doped SWCNTs- based gas sensors were studied in the hydrogen atmosphere.

In Figure 7, the temperature characteristics of three SWCNTsbased gas sensors are illustrated among $150-400^{\circ} \mathrm{C}$ to $100 \mu \mathrm{L} / \mathrm{L}$ hdrogen. On the one hand, three different SWCNTs-based gas sensors present the same trend of temperature characteristics: curves climb at first with the increase of the temperature and decrease after reaching the optimal operating temperature. This might be because when heated to a certain temperature, the surface of SWCNTs nanomaterials is occupied by oxygen atoms released from the air, which increases the surface conductance of the materials and, thus, shows a decrease in resistance. On the other hand, after modifying SWCNTs-based nanomaterials with Pd nanoparticles, the optimal operating temperature dropped sharply by $125^{\circ} \mathrm{C}$, and the peak response of Pd-doped SWCNTsbased gas sensor is 10.1 and 3.2 times higher than that of the unloaded and carboxylated SWCNTs-based gas sensors under the respective optimal operating temperature, respectively.

Concentration characteristics were tested in $0-500 \mu \mathrm{L} / \mathrm{L}$ hydrogen at $275^{\circ} \mathrm{C}$, as shown in Figure 8. As the gas concentration rises, the growth rate of the response value of all three gas sensors slows down and gradually becomes 


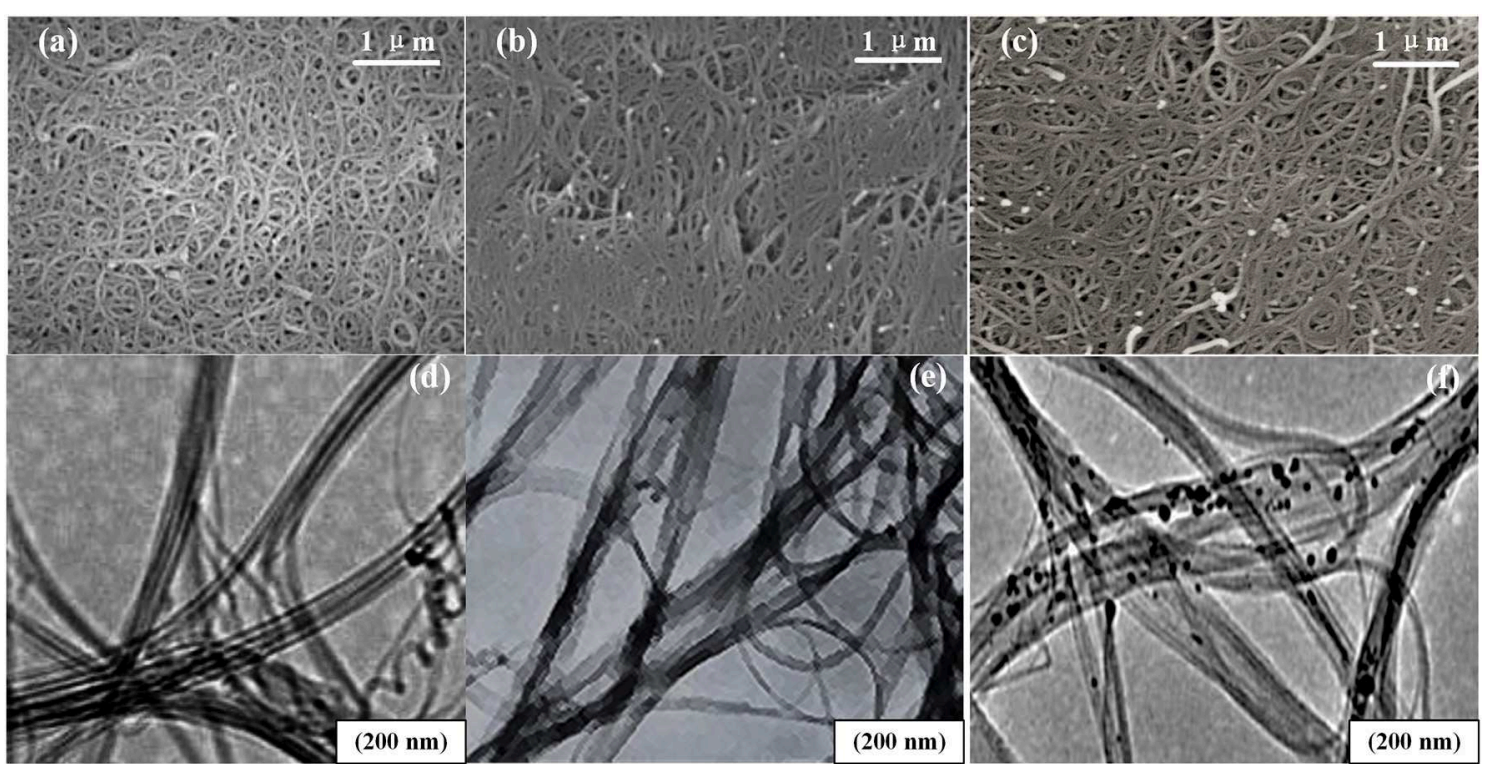

FIGURE 6 | The morphology of nanomaterials. (a-c) The SEM images of the unloaded, the carboxylated, and the Pd-doped SWCNTs. (d-f) The TEM images of the unloaded, the carboxylated, and the Pd-doped SWCNTs.

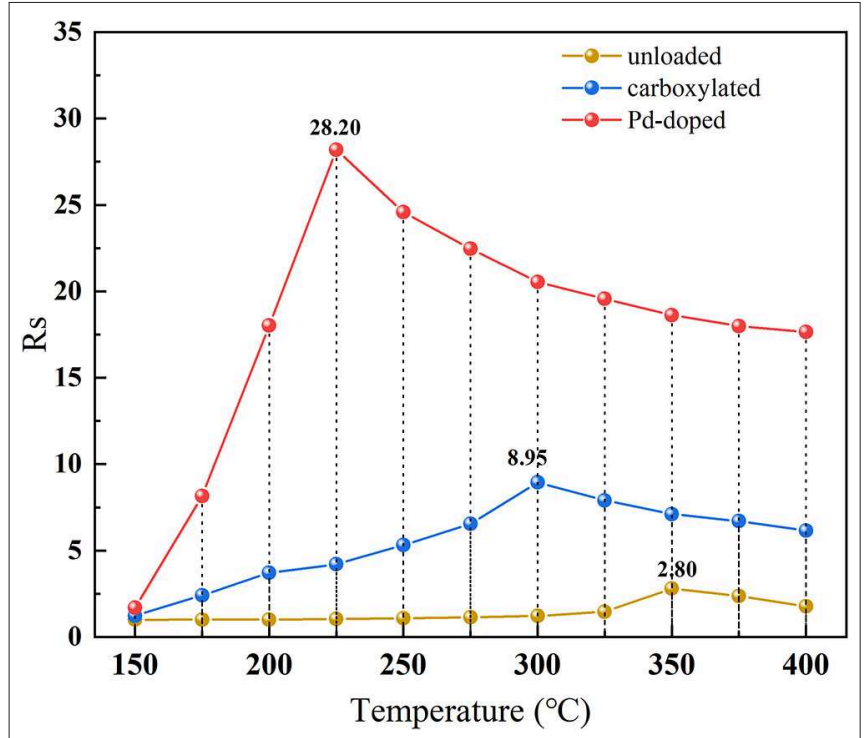

FIGURE 7 | The temperature characteristics of the unloaded, carboxylated, and 3.07 wt\% Pd-doped SWCNTs-based gas sensors.

saturated. However, 3.07 wt\% Pd-doped SWCNT-based gas sensor has better gas sensing performance in an extreme high gas concentration atmosphere. When the concentration of hydrogen is $500 \mu \mathrm{L} / \mathrm{L}$, the gas sensing response of Pd-doped SWCNTsbased gas sensor is about 33.79, which is almost 14 and 3.7 times higher than the gas sensing response of the unloaded and the carboxylated SWCNTs-based gas sensors, respectively.

In Figure 8, $R_{s}$ is linearly related to the concentration in the respective low concentration ranges $(1-30 \mu \mathrm{L} / \mathrm{L})$, and the fitting function and corresponding $R^{2}$ (linearity) is shown in Table 1. Assuming that when hydrogen is $1 \mu \mathrm{L} / \mathrm{L}$, the gas sensing response

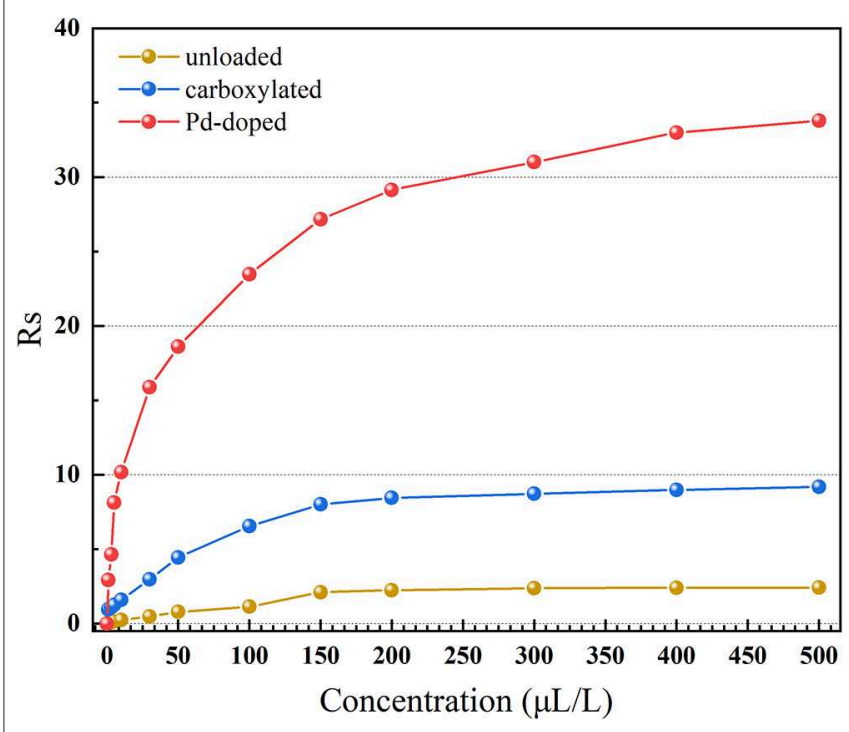

FIGURE 8 | The concentration characteristics of the unloaded, carboxylated, and 3.07 wt\% Pd-doped SWCNTs-based gas sensors.

is not lower than 1 , the corresponding gas sensor is able to reach the detecting limitation (International Standard IEC 60599: 2015, 2015; Tang et al., 2017). Results are shown in Table 1. SWCNTsbased gas sensors with or without functionalization all show perfect linearity, while only the Pd-doped SWCNTs-based gas sensor can meet the requirement of detecting limitation.

Figure 9 presents the response and recovery time characteristics of three gas sensors which were tested at $275^{\circ} \mathrm{C}$ to $50 \mu \mathrm{L} / \mathrm{L}$ hydrogen. The experiment was repeated three times: $50 \mu \mathrm{L} / \mathrm{L}$ hydrogen was injected at 50,800 , and $1,550 \mathrm{~s}$, and was exhausted at $450,1,200$, and $1,950 \mathrm{~s}$. It is obvious that 
TABLE 1 | The fitting function, linearity and detecting limitation of three SWCNTs-based gas sensors to hydrogen.

\begin{tabular}{llcc}
\hline Sensor units & The fitting function & $\mathbf{R}^{2}$ & Detection limit \\
\hline Unloaded & $y=0.0148 x+0.0574$ & 0.994 & $\mathbf{x}$ \\
Carboxylated & $y=0.0706 x+0.8921$ & 0.9999 & $\mathbf{x}$ \\
Pd-doped & $y=1.2757 x+0.9729$ & 0.9697 & $\checkmark$
\end{tabular}

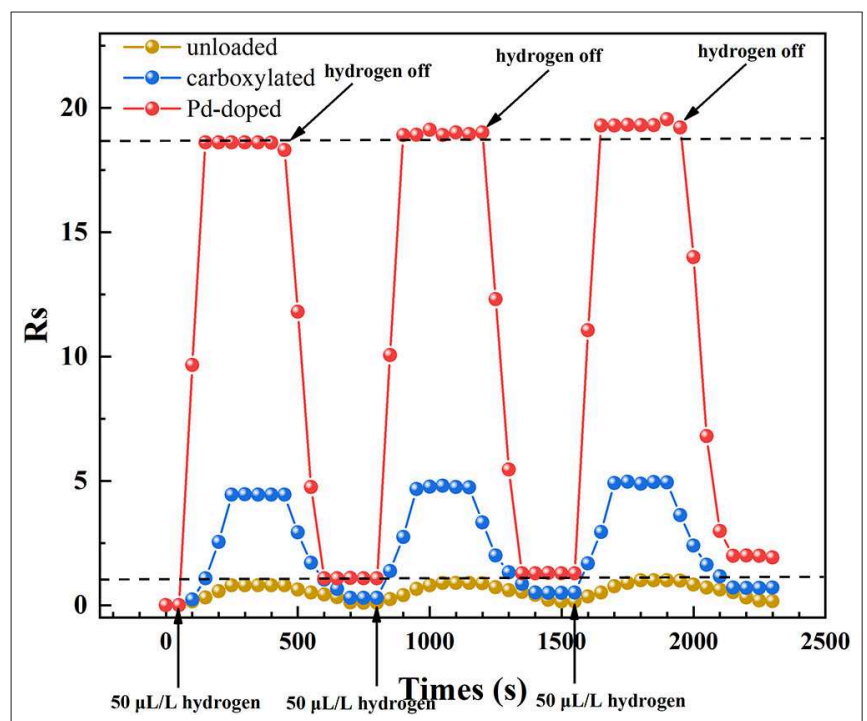

FIGURE 9 | The gas response and recovery time characteristics of three different SWCNTs-based gas sensors.

both response and recovery time of Pd-doped SWCNTs-based gas sensor are shorter than the other two. The response time and recovery time of Pd-doped SWCNTs-based gas sensor are about 100 and $150 \mathrm{~s}$. What's more, the gas sensing response value of the three gas sensors is slightly increased after the third time test, which might be because of the incomplete exhaust each time, resulting in the presence of more hydrogen than expected in the final gas chamber. The recovery process of gas sensing materials is a dynamic process of adsorption and desorption between the molecules of the test gas, hydrogen, and nanotubes. If the hydrogen molecules in the gas chamber are not exhausted cleanly, a small amount of hydrogen molecules will still be adsorbed with the active sites on the surface of the nanotube at high temperatures, shown as the hydrogen atoms, so that there will still be less electron exchange in the gas sensing material nanotubes.

\section{Gas Sensing Mechanism}

Obviously, after decorating SWCNTs with Pd nanoparticles, the gas sensing performance to hydrogen was remarkably enhanced. This is attributed to the enhanced electrical property toward molecular hydrogen compared with the undecorated SWCNTs. The gas sensing reaction is the interactions between $\mathrm{H}_{2}, \mathrm{Pd}$, and the nanotubes. After heating, hydrogen molecules dissociate faster into atomic hydrogen on Pd surface, resulting in the dissolution of atomic hydrogen in $\mathrm{Pd}$ with high solubility, consequently decreasing the work function of $\mathrm{Pd}$, that is shown in the formula (1). The lowering of the work function of Pd leads to faster and easier electron transfer from Pd to SWCNTs, reducing the number of hole-carries in the p-type nanotubes and the value of conductance.

When the Pd-doped SWCNTs-based gas sensor was in a low hydrogen atmosphere, the reason why it can reverse and auto recover is because of the oxygen in the air and on the surfaces, which is represented as the formulas (2) and (3) (Mandelis et al., 1993; Collins, 2000; Kong, 2000).

$$
\begin{aligned}
H_{2} \rightarrow 2 H_{\text {atom on surfaces }} H_{\text {atom on surfaces }} & \rightarrow H_{\text {atom in Pd }} \\
\mathrm{O}_{2}+2 \mathrm{H}_{\text {atom on surfaces }} & \rightarrow 2 \mathrm{OH} \\
\mathrm{OH}+\mathrm{H}_{\text {atom on surfaces }} & \rightarrow \mathrm{H}_{2} \mathrm{O}
\end{aligned}
$$

\section{CONCLUSION}

In this paper, we prepared the carboxylated and $3.07 \mathrm{wt} \% \mathrm{Pd}-$ doped SWCNTs-based nanomaterials based on the unloaded SWCNTs, and three different SWCNTs-based planar gas sensors were fabricated and tested in hydrogen to study their gas sensing properties. Results show that functionalized SWCNTs-based gas sensor with Pd nanoparticles present the best gas sensing performance, and has the lowest optimal operating temperature $\left(225^{\circ} \mathrm{C}\right)$ and the highest gas sensing response to $500 \mu \mathrm{L} / \mathrm{L}$ hydrogen at $275^{\circ} \mathrm{C}\left(R_{s} \cong 33.79\right)$. The gas response and recovery time of Pd-doped SWCNTs-based gas sensor are both $50 \mathrm{~s}$ shorter than those of the unloaded and carboxylated SWCNTs-based gas sensors. This was because the Pd doping lowers the work function and enhances the electrical property toward molecular hydrogen. Results could assist the development of novel SWCNTs-based gas sensors for fault characteristic gases detection in the high-voltage electrical transformers.

\section{DATA AVAILABILITY STATEMENT}

The datasets generated for this study are available on request to the corresponding author.

\section{AUTHOR CONTRIBUTIONS}

ST designed the experiment, finished the experiment, data collected and analyzed, and wrote the paper. WC helped correct the paper. HZ helped design and finished the experiment. ZS helped collect the data. YL helped correct the paper. YW helped correct the paper.

\section{FUNDING}

This work was supported by the National Science Foundation of China (Grant No. U1766217), Fundamental Research Funds for the Central University (No. 2019CDJGFCL001), and State Grid Corporation of China Science and Technology Project (52110418000Q). 


\section{REFERENCES}

Beitollahi, H., Movahedifar, F., Tajik, S., and Jahani, S. (2018). A review on the effects of introducing CNTs in the modification process of electrochemical sensors. Electroanalysis 31, 1195-1203. doi: 10.1002/elan.201800370

Chen, R., Franklin, N., Kong, J., Cao, J., Tombler, T., Zhang, Y., et al. (2001). Molecular photodesorption from single-walled carbon nanotubes. Appl. Phys. Lett. 79, 2258-2260. doi: 10.1063/1.1408274

Collins, P. G. (2000). Extreme oxygen sensitivity of electronic properties of carbon nanotubes. Science 287, 1801-1804. doi: 10.1126/science.287.5459.1801

Dai, H., Kong, J., Zhou, C., Franklin, N., Tombler, T., Cassell, A., et al. (1999). Controlled chemical routes to nanotube architectures, physics, and devices. J. Phys. Chem. B. 103, 11246-11255. doi: 10.1021/jp992328o

Han, T., Nag, A., Mukhopadhyay, S. C., and Xu, Y. (2019). Carbon nanotubes and its gas-sensing applications: a review. Sens. Actuators A Phys. 291, 107-143. doi: 10.1016/j.sna.2019.03.053

International Standard IEC 60599: 2015 (2015). Mineral Oil-Filled Electrical Equipment in Service-Guide on the Interpretation of Dissolved and Free Gases Analysis. International Electrotechnical Commission.

Kong, J. (2000). Nanotube molecular wires as chemical sensors. Science 287, 622-625. doi: 10.1126/science.287.5453.622

Kong, J., Chapline, M. G., and Dai, H. (2001). Functionalized carbon nanotubes for molecular hydrogen sensors. Adv. Mater. 13, 1384-1386. doi: 10.1002/1521-4095(200109)13:18<1384::AID-ADMA1384>3.0.CO;2-8

Kong, J., Soh, H., Cassell, A., Quate, C., and Dai, H. (1998). Synthesis of single single-walled carbon nanotubes on patterned silicon wafers. Nature 395, 878-881. doi: 10.1038/27632

Lone, M., Kumar, A., Husain, S., Singh, R. C., Zulfequar, M., and Husain, M. (2018). Fabrication of sensitive SWCNT sensor for trace level detection of reducing and oxidizing gases $\left(\mathrm{NH}_{3}\right.$ and $\left.\mathrm{NO}_{2}\right)$ at room temperature. Phys. E Low Dimens. Syst. Nanostruct. 108, 206-214. doi: 10.1016/j.physe.2018.11.020

Mandelis, A., Christofides, C., and Winefordner, J. D. (1993). Physics, Chemistry and Technology of Solid State Gas Sensor Devices. New York, NY: Wiley.

Naje, A., Ibraheem, R., and Ibrahim, F. (2016). Parametric analysis of $\mathrm{NO}_{2}$ gas sensor based on carbon nanotubes. Photonic Sens. 6, 153-157. doi: 10.1007/s13320-016-0304-1

Pitroda, J., Jethwa, B., and Dave, S. K. (2016). A critical review on carbon nanotubes. Int. J. Construct. Res. Civil Eng. 2, 36-42. doi: 10.20431/2454-8693.0205007

Poonia, M., Manjuladevi, V., Gupta, R., Gupta, S. K., Singh, J., Agarwal, P., et al. (2015). Ultrathin films of single-walled carbon nanotubes: a potential methane gas sensor. Sci. Adv. Mater. 7, 455-462. doi: 10.1166/sam.20 15.1989

Rana, M., Dauda, S., Mohd, R., Mohd, A., Jarin, S., and Tomal, A. (2017). A review on recent advances of CNTs as gas sensors. Sens. Rev. 37, 127-136. doi: 10.1108/SR-10-2016-0230

Robertson, J. (2004). Realistic applications of CNTs. Mater. Today 7, 46-52. doi: 10.1016/S1369-7021(04)00448-1
Tabtimsai, C., Keawwangchai, S., Wanno, B., and Ruangpornvisuti, V. (2011). Gas adsorption on the $\mathrm{Zn}$-, $\mathrm{Pd}$ - and Os-doped armchair $(5,5)$ single-walled carbon nanotubes. J. Mol. Model. 18, 351-358. doi: 10.1007/s00894-011-1047-y

Tang, S., Chen, W., Xu, L., and Gao, T. (2017). Fabrication of Ag-doped ZnO nanoparticle gas sensor and its application in detection of CO. Nanosci. Nanotechnol. Lett. 9, 214-219. doi: 10.1166/nnl.2017.2313

Wang, C., Yin, L., Zhang, L., Xiang, D., and Gao, R. (2010). Metal oxide gas sensors: Sensitivity and influencing factors. Sensors 10, 2088-2106. doi: 10.3390/s100302088

Wang, J., Zhou, Q., Lu, Z., Wei, Z., and Zeng, W. (2019). Gas sensing performances and mechanism at atomic level of Au-MoS 2 microspheres. Appl. Surf. Sci. 490, 124-136. doi: 10.1016/j.apsusc.2019.06.075

Wei, Z., Zhou, Q., Lu, Z., Xu, L., Gui, Y., and Tang, C. (2019). Morphology controllable synthesis of hierarchical $\mathrm{WO}_{3}$ nanostructures and $\mathrm{C}_{2} \mathrm{H}_{2}$ sensing properties. Phys. E Low Dimens. Syst. Nanostruct. 109, 253-260. doi: 10.1016/j.physe.2019.01.006

Yoon, B., Choi, S.-J., Swager, T., and Walsh, G. (2018). Switchable single-walled carbon nanotube-polymer composites for $\mathrm{CO}_{2}$ sensing. ACS Appl. Mater. Interf. 10, 33373-33379. doi: 10.1021/acsami.8b11689

Zaporotskova, I., Boroznina, N., Parkhomenko, Y., and Kozhitov, L. (2017) Carbon nanotubes: sensor properties. A review. Modern Electr. Mater. 2, 95-105. doi: 10.1016/j.moem.2017.02.002

Zhang, X., Cui, H., Gui, Y., and Tang, J. (2017). Mechanism and application of carbon nanotube sensors in $\mathrm{SF}_{6}$ decomposed production detection: a review. Nanoscale Res. Lett. 12:177. doi: 10.1186/s11671-017-1945-8

Zhou, Q., Umar, A., Sodki, E., Amine, A., Xu, L., Gui, Y., et al. (2017a). Fabrication and characterization of highly sensitive and selective sensors based on porous NiO nanodisks. Sens. Actuators B Chem. 259, 604-615. doi: 10.1016/j.snb.2017.12.050

Zhou, Q., Xu, L., Umar, A., Chen, W., and Kumar, R. (2017b). Pt nanoparticles decorated $\mathrm{SnO}_{2}$ nanoneedles for efficient $\mathrm{CO}$ gas sensing applications. Sensors and Actuators B Chem. 256, 656-664. doi: 10.1016/j.snb.2017.09.206

Zhou, T., Liu, X., Zhang, R., Wang, L., and Zhang, T. (2018). Constructing hierarchical heterostructured $\mathrm{Mn}_{3} \mathrm{O}_{4} / \mathrm{Zn}_{2} \mathrm{SnO}_{4}$ materials for efficient gas sensing reaction. Adv. Mater. Interf. 5:1800115. doi: 10.1002/admi.201800115

Conflict of Interest: The authors declare that the research was conducted in the absence of any commercial or financial relationships that could be construed as a potential conflict of interest.

Copyright $\odot 2020$ Tang, Chen, Zhang, Song, Li and Wang. This is an open-access article distributed under the terms of the Creative Commons Attribution License (CC $B Y)$. The use, distribution or reproduction in other forums is permitted, provided the original author(s) and the copyright owner(s) are credited and that the original publication in this journal is cited, in accordance with accepted academic practice. No use, distribution or reproduction is permitted which does not comply with these terms. 